\title{
Research Paper: Comparison Between Switching and Creativity Among Bilingual and Monolingual Children
}

\author{
Rahim Yousefi ${ }^{1},{ }^{*}$ Mehran Soleimani ${ }^{1}$, Samira Ghazanfariyanpoor ${ }^{1}$
}

1. Department of Psychology, Faculty of Education and Psychology, Azarbaijan Shahid Madani University, Tabriz, Iran.

Citation: Yousefi R, Soleimani M, Ghazanfariyanpoor S. [Comparison Between Switching and Creativity Among Bilingual and Monolingual Children (Persian)]. Archives of Rehabilitation. 2017; 18(1):1-12. https://doi.org/ 10.21859/jrehab-18011

https://doi.org/10.21859/jrehab-18011

Received: 15 Oct. 2016 Accepted: 12 Jan. 2017
Keywords: Cognition, Attention, Stroop test, Creativity, Multilingualism

\section{ABSTRACT}

Objective Several studies have reported that bilingualism may affect cognitive processes. Second language acquisition takes place in a variety of ways. However, considering the fact that language training courses provided by institutes are expanding at a blistering pace, the effects of foreign language learning through the medium of language schools deserves a separate line of investigation in the realm of research pertaining to bilingualism. This study aimed at probing the effect of language learning on children by comparing the switching and creativity levels of those who undertook advanced English classes and others who did not.

Methods \& Materials A cross-sectional, causal-comparative study was conducted. The population of this research included all monolingual and bilingual children in the age group of 13-15 years in Isfahan in the year 2015. Sixty-four children pursuing advanced levels of English (level RECE and REACH from the center of Iranian language and equivalent levels in other schools) were part of the intervention group, and 51 monolingual children of the same age group were selected as participants in the control group. The purposive sampling method was used. In order to evaluate and measure children's creativity in the monolingual and bilingual groups, the Torrance creativity questionnaire was used. The classic Stroop test D-KEFS CW was used to measure the ability of switching of monolingual and bilingual children. The data obtained was analyzed by way of descriptive statistics and multivariate analysis of variance (MANOVA), using SPSS software (version 22).

Results The results showed that there is a significant difference between children belonging to bilingual and monolingual groups in terms of switching $(P<0.05)$. Bilingual children acted better in switching assignments than their monolingual counterparts. Also, there was a significant difference between the two groups with regard to the scores of fluency, flexibility, and elaboration $(\mathrm{P}<0.001)$, which are components of creativity. There was no significant difference between children in the two groups in terms of originality $(P>0.01)$.

Conclusion In accordance with the results of the study, it can be concluded that learning English through educational institutions, and at an advanced level, significantly increases the switching capability in children as well as their scores across the three components of creativity (fluency, flexibility, and elaboration). Therefore, the role of second language acquisition should be highlighted because of its contribution to children's creativity and ability to switch. In general, better performances of children are attributed to the capability of switching languages, specific cognitive mechanisms used in the two systems of languages, their familiarity with a new culture and customs while learning a new language, the intensive training sessions as well as the special atmosphere prevailing in the classes.

\section{* Corresponding Author:}

Mehran Soleimani, PhD

Address: Department of Psychology, Faculty of Education \& Psychology, Azarbaijan Shahid Madani University, Tabriz, Iran.

Tel: +98 (914) 3632650

E-Mail: mehransoleymani21@gmail.com 


\title{
مقايسه تغيير توجه و خلاقيت در كودكان دوزبانه و تكزبانه
}

\author{
رحيم يوسفى'، "مهران سليمانى'، سميرا غضنفريانيور' \\ ا - كروه روانشناسي، دانشكده علوم تربيتى ورواتشناسى، دانشَّاه شهيد مدنى آذربايجان، تبريز، ايران.
}

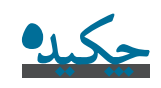

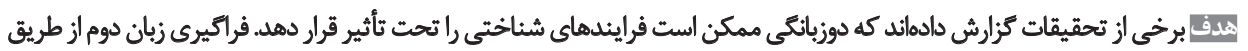

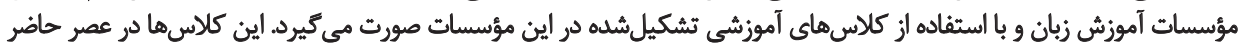

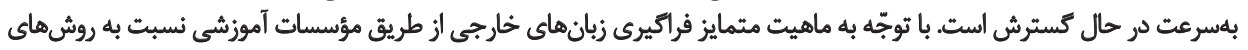

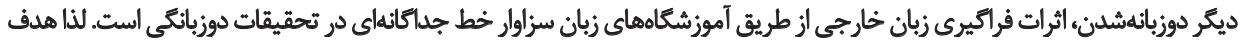

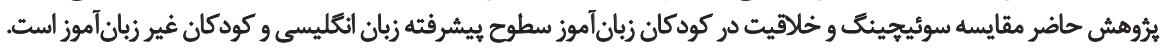

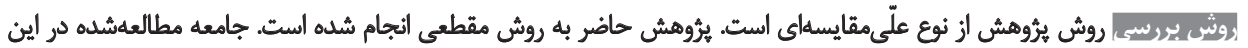

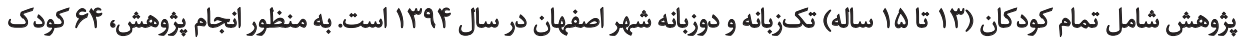

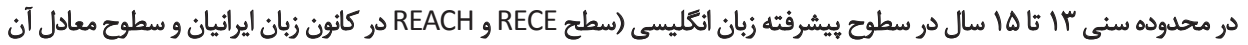

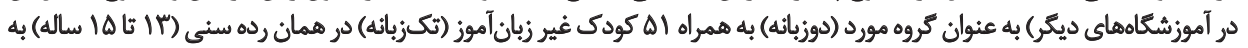

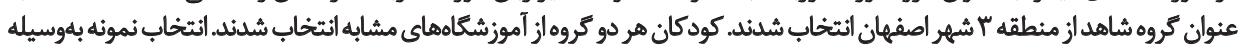

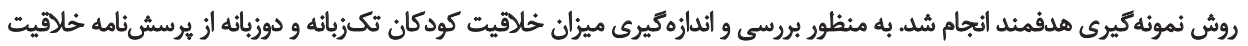

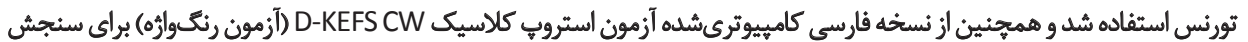

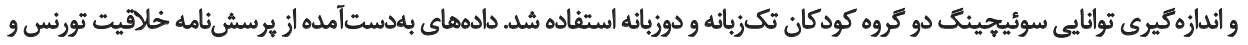

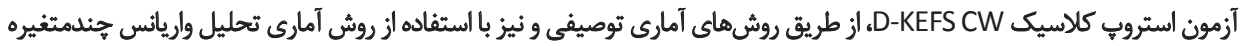

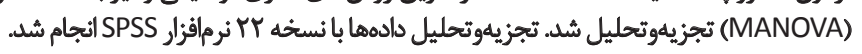

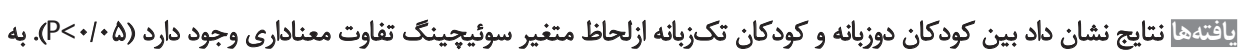

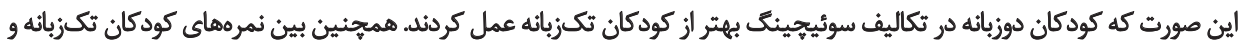

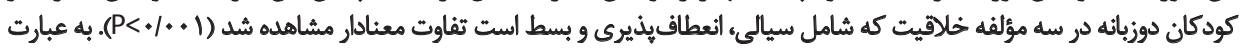

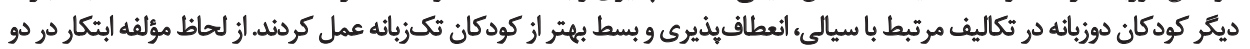

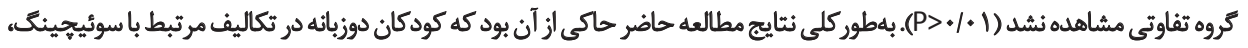

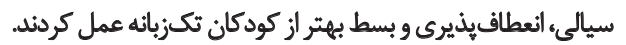

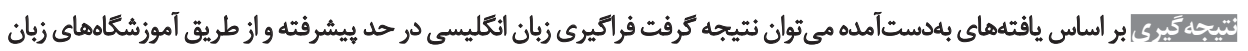

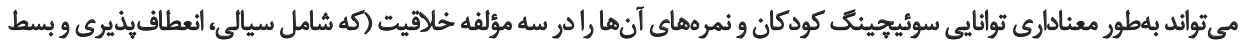

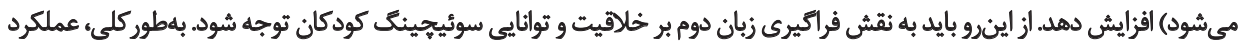

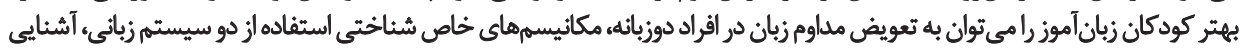

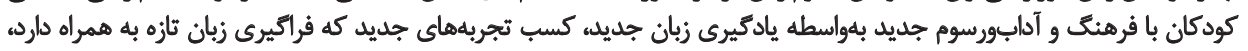

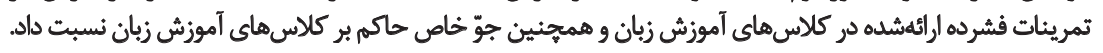

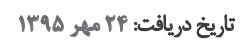

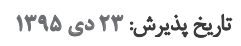

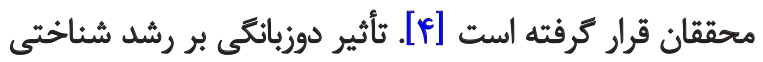

مقدمه

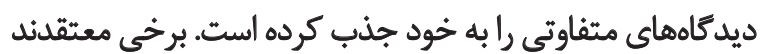

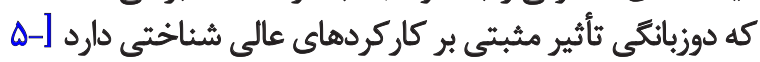

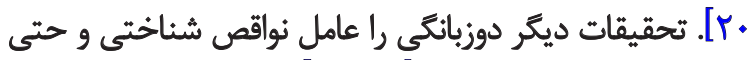

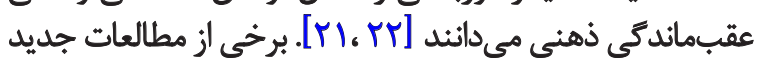

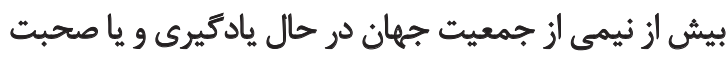

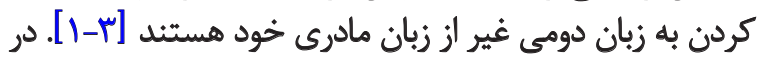
سالهاى اخير رابطه زبان و شناخت درئ درئ دوزبانهها مورد توجه

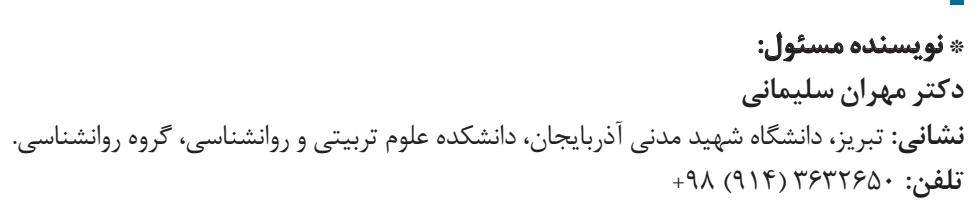

رايانامه: mehransoleymani21@gmail.com 
را براى كنترل اجرايى آنها دربر داشته باشد [FA، F9، F] والترز" (F) (T) مدعى بود انعطافيذيرى خاصى كه دوزبانهها

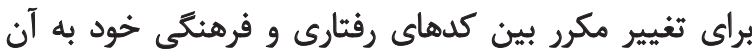

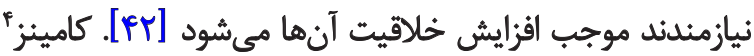
(19VV)

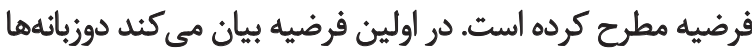

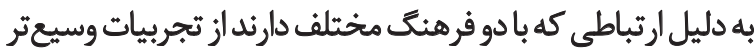

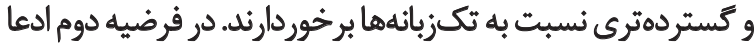

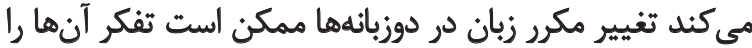

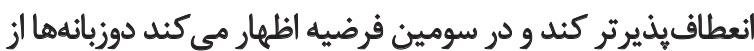

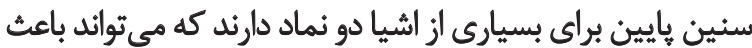

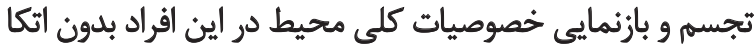

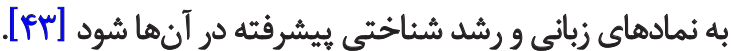

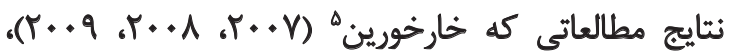

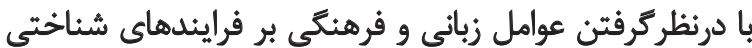

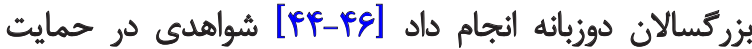

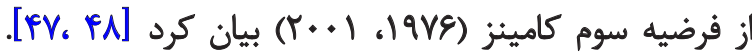

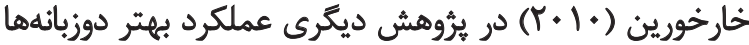

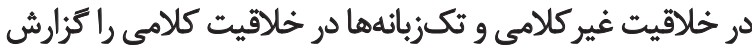

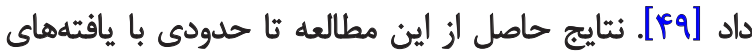

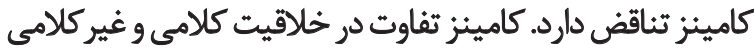

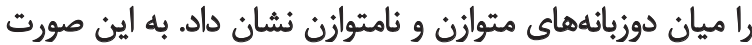

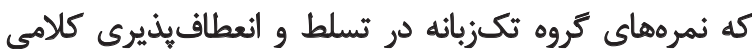

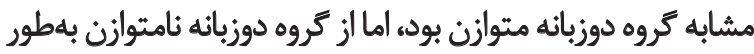

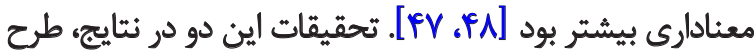

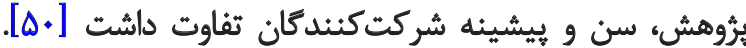

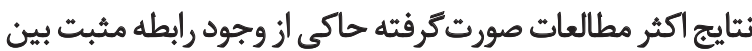

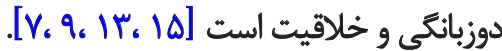

مطالعات انجامشده در نمونه و روش و ويزگگىها بسيار متنوع

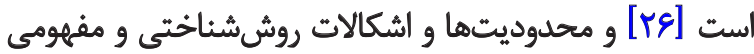

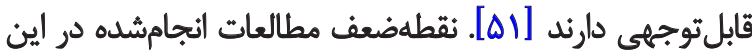

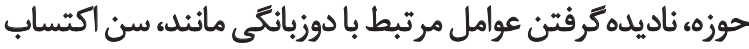

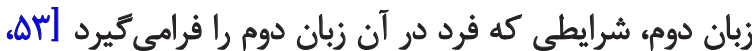

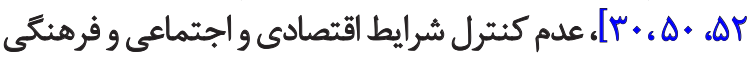

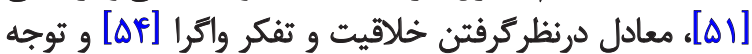

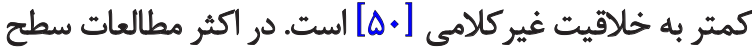

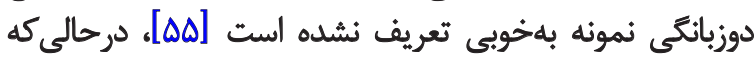

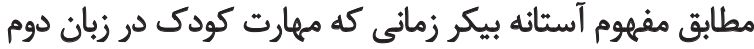

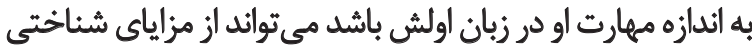

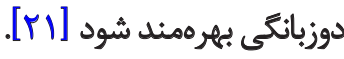

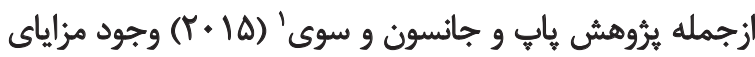

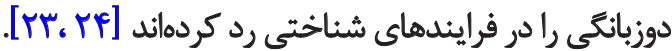

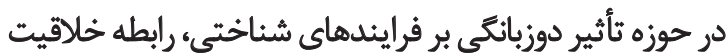

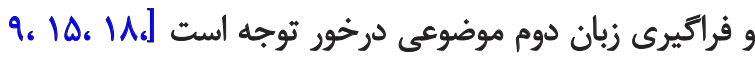

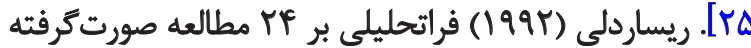

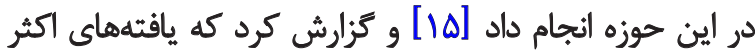

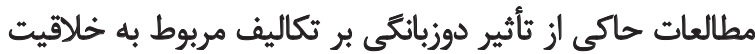

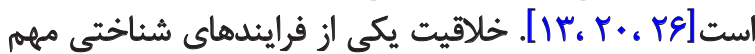

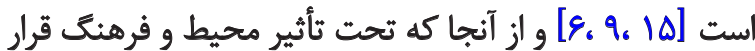

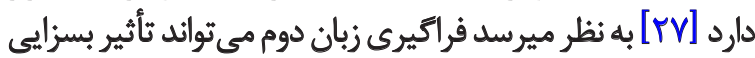

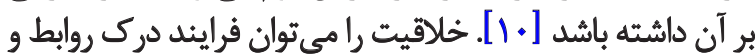

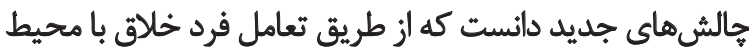

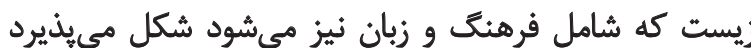

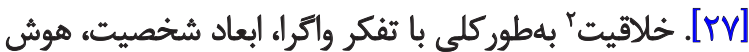

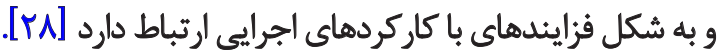
تحقيقات ثأثيرات حالتهاى مختلف كنترل شناختى بر

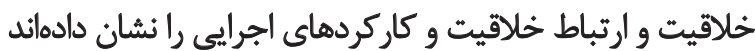

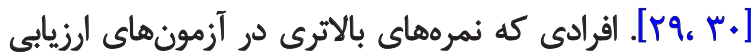

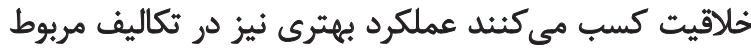

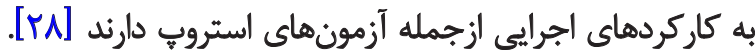

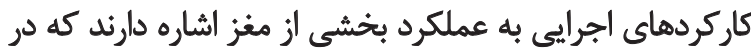

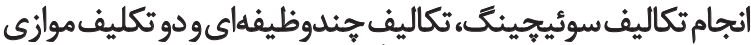

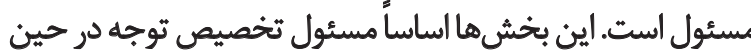

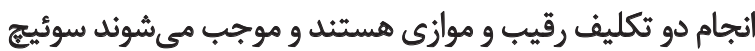

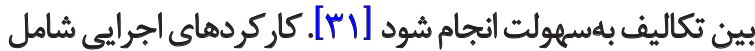

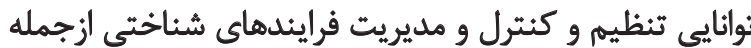

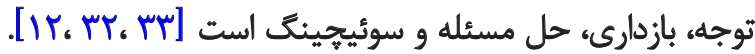

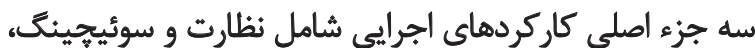

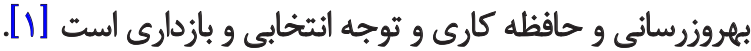

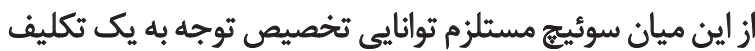

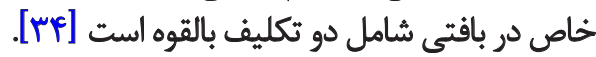

كنترل اجرايى و كاركردهاى اجرايى مسئول مديريت دو سيستم

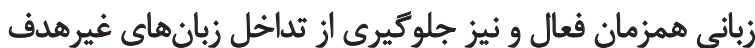

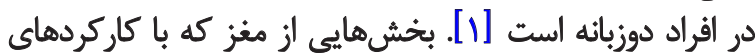

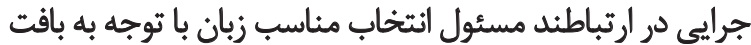

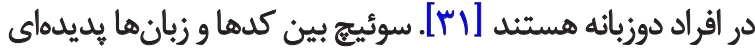

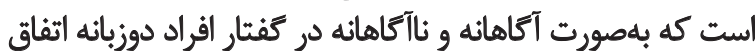

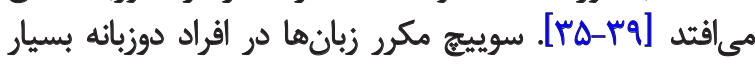
شبيه تكاليف و تمرينهاى سوئيج و دو وظيفهائ است كه برائ برائ

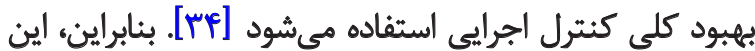

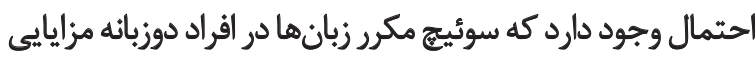




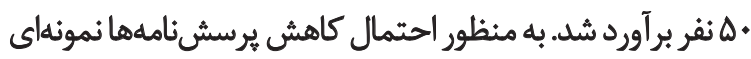

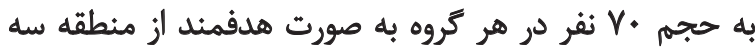

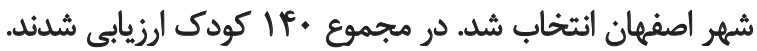

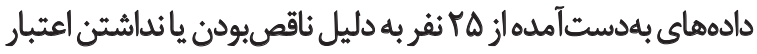

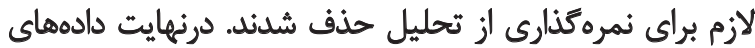

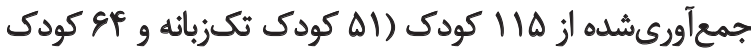

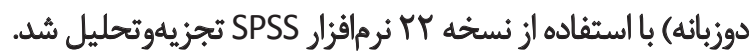

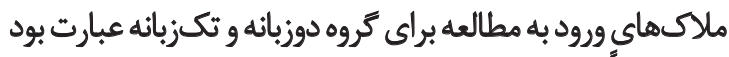

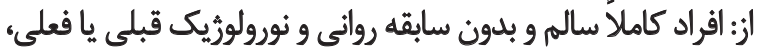

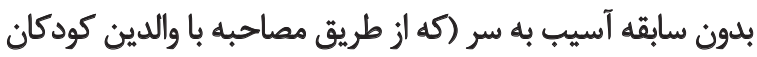

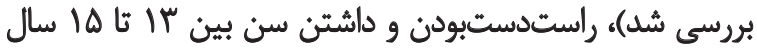

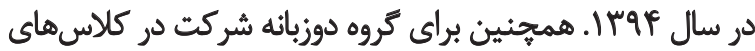

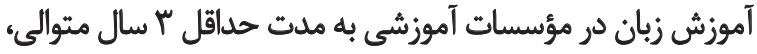

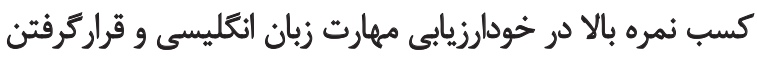

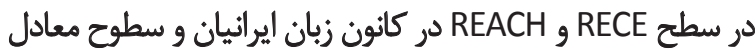

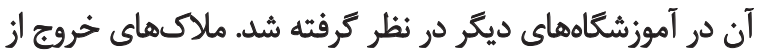

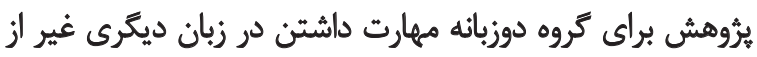

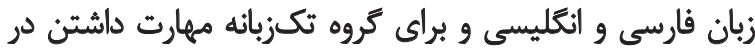

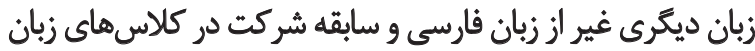

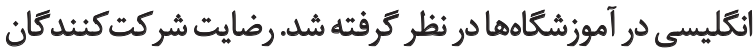

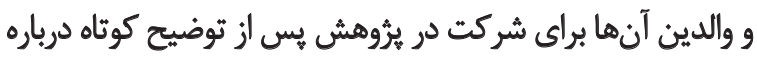

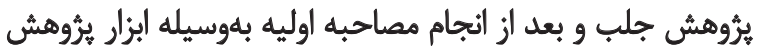

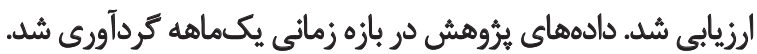

در اين بُروهش از برسشنامه ويرّكى هاى جمعيت شناختى و

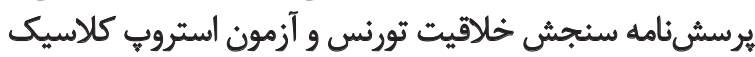

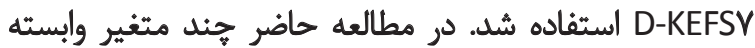

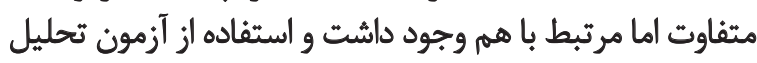

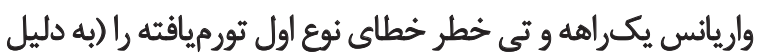

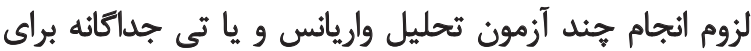

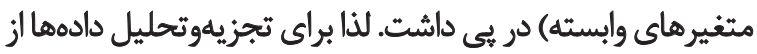

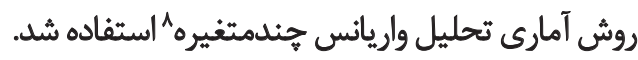

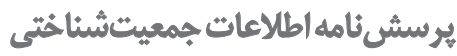

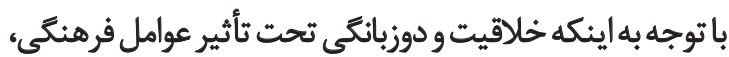

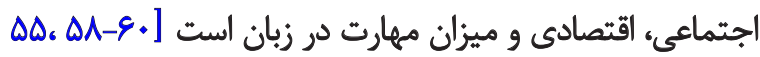
[\&F،

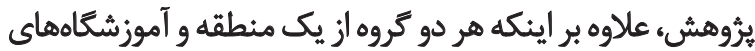

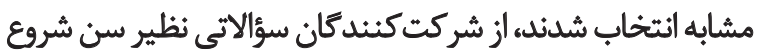

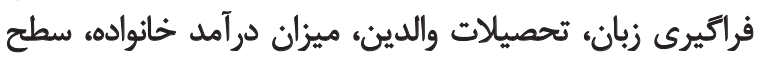

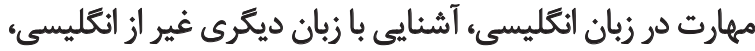

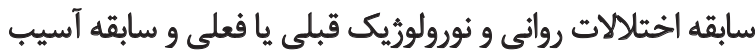

7. Delise Kaplan Executive Function System 8. MANOVA
كامينز (19VV) به شرح مفهوم آستانه برداخت و بيان كران كرد

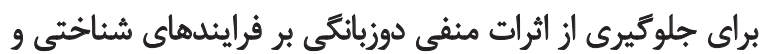

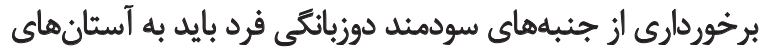

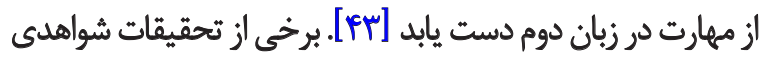

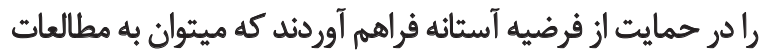

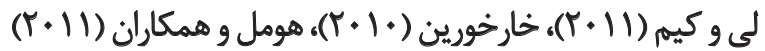

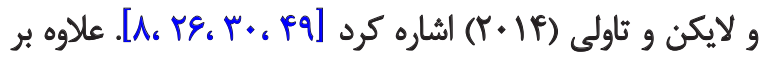

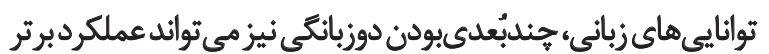

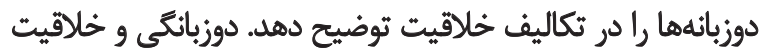

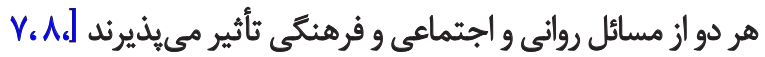

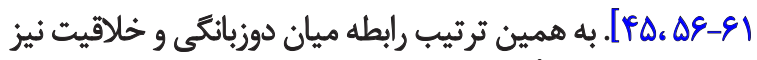

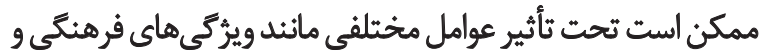

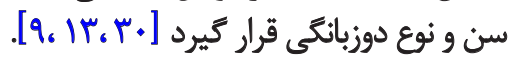

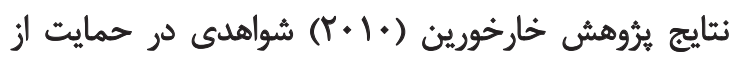

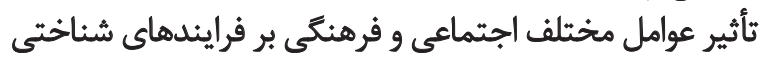

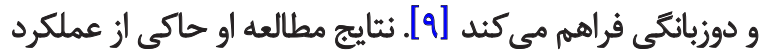

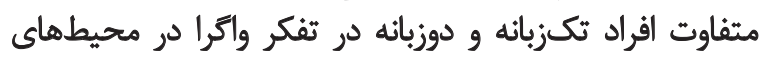

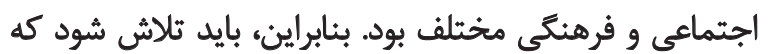

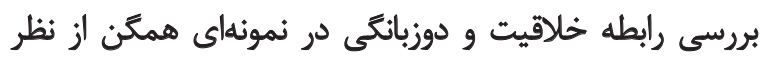

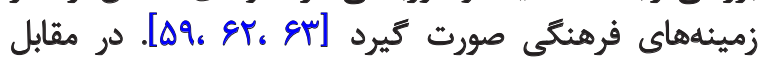

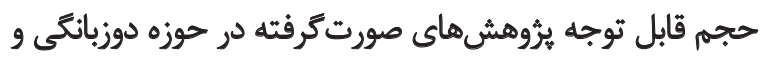

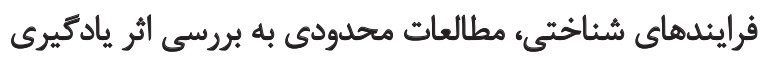

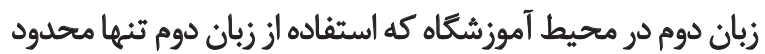

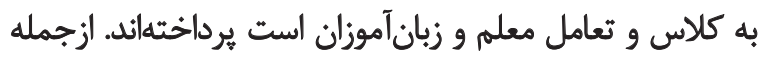

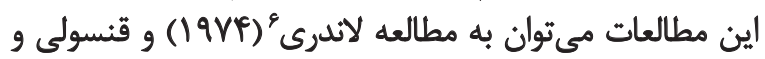

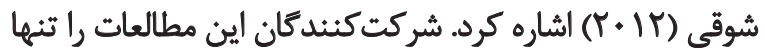

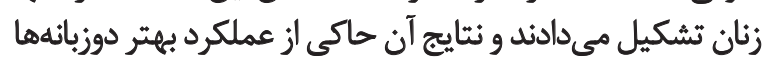
در تكاليف خلاقيت بود [QT)]

با توجه به موارد ذكرشده و اين واقعيت كه مطالعات انجامشده

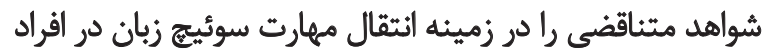

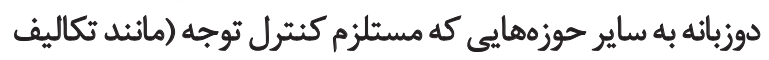

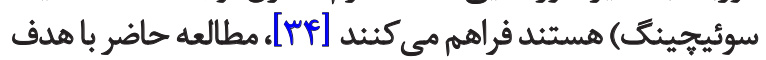

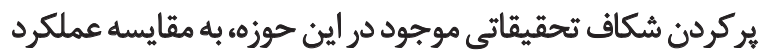

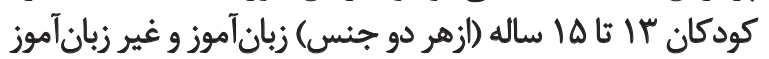

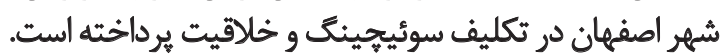

$$
\text { ووشُ بروسي }
$$

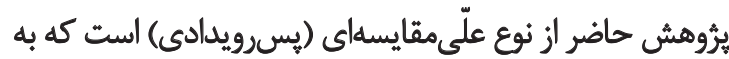

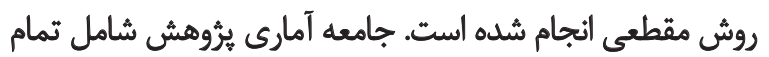

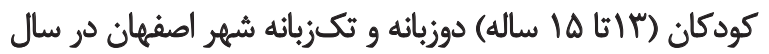

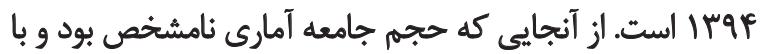

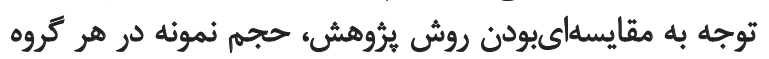

6. Landry 
سه كَزينهاي است و هر كَينه نشاندهنده ميزان خلاقيت بايين،

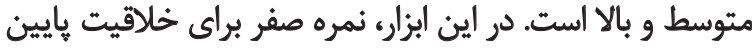

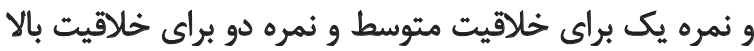

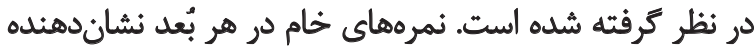

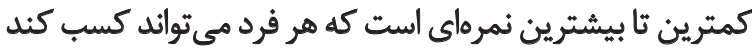

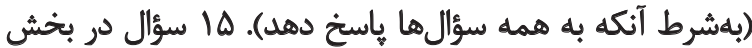

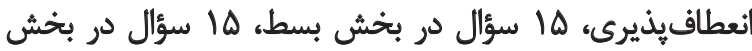
سيالى و ها سؤال در بخش ابتكار مطرح مي دشود.

عابدى (1999) ضريب اعتبار بخشهاى سيالى، ابتكار،

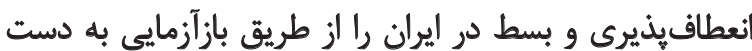

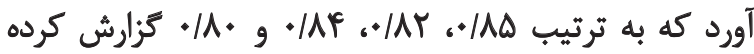

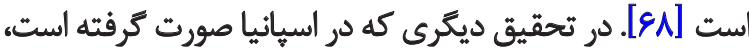

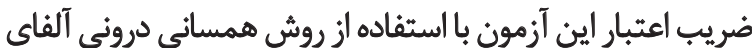

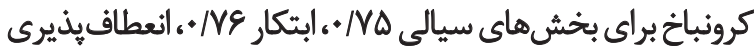

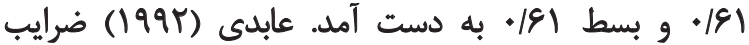

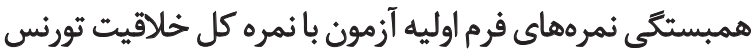

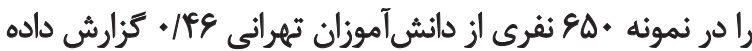

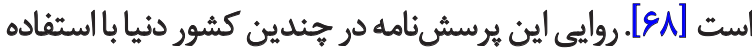

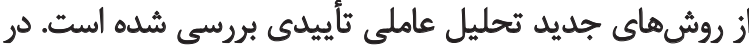

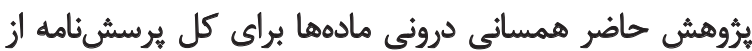

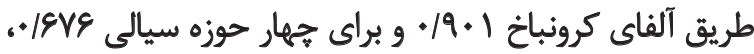

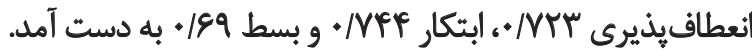

دستكاه

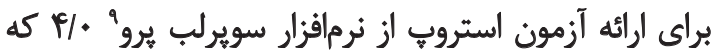

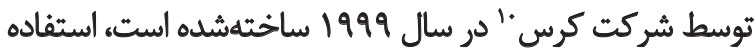

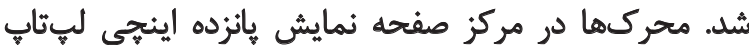

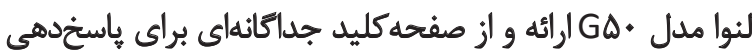

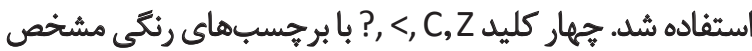

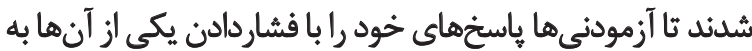

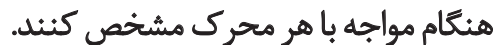

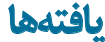

اطلاعات و يافتههاى جمعيتشناختى گروه نمونه مانئد سن

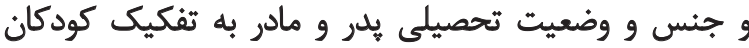

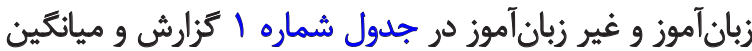

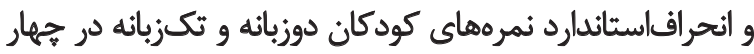
حوزه خلاقيت در جدول شماره ب ارائه شده است.

به منظور آزمودن فرضيه يرؤوهش مبنى بر وجود تفاوت

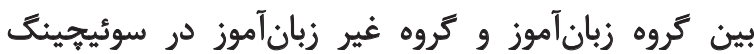
و جهار خردهمقياس خلاقيت از روش زئ تحليل واريانس

9. Super Lab Pro

10. Cerdus corporation

\section{به سر برسيله شلد. \\ آزمون استروب كلاسيك D-KEFES CW}

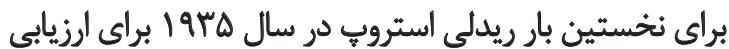

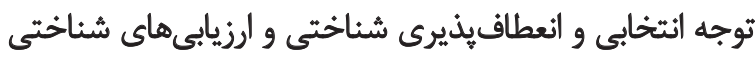

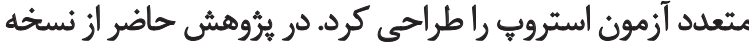

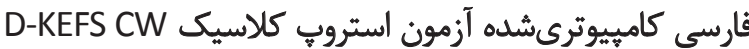

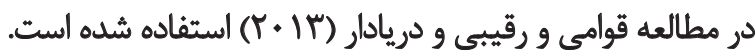

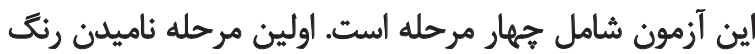

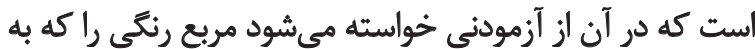

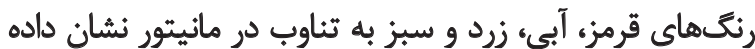

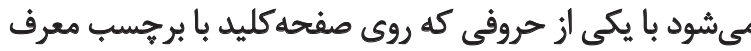
مشخص شده است نشان دهد.

دومين مرحله خواندن كلمه است. در اين مرحله از آزمودنى

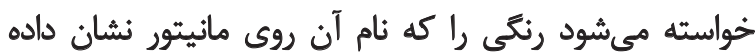

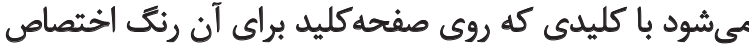

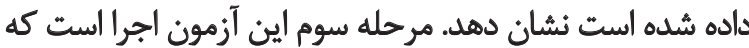

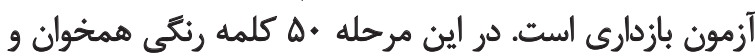

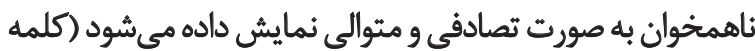

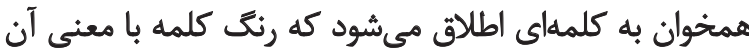

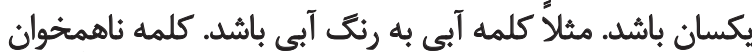

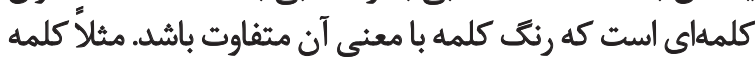

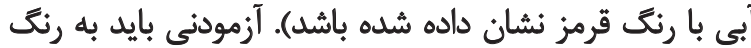

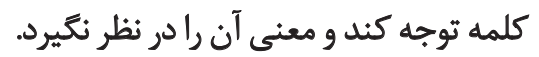

مرحله جهارم آزمون سوئيج و بازدارى است. در اين مرحله

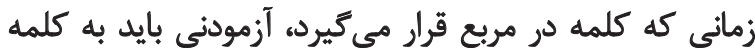

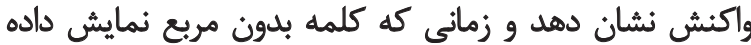

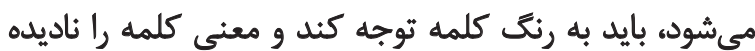

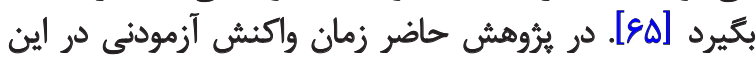

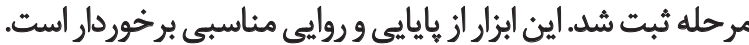

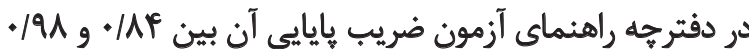

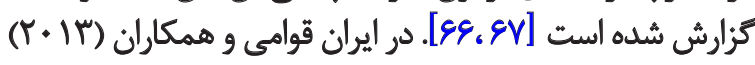

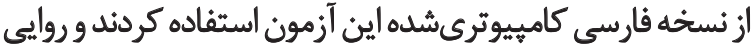

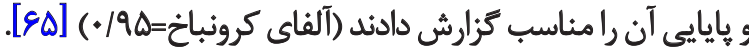

$$
\text { برسش نامه سنجش خلاقيت ثورنس }
$$

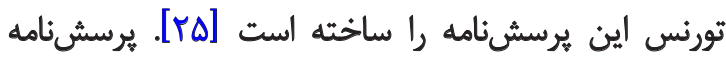

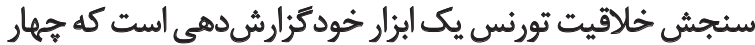

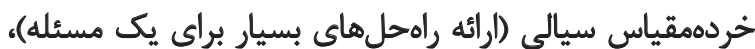

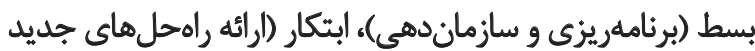

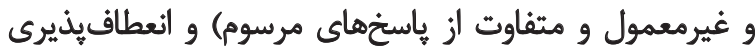
(جداشدن از الكوهاى قديمى تفكر و استفاده از شيوههاى جداى جديد

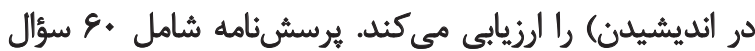




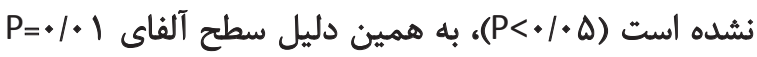

ث

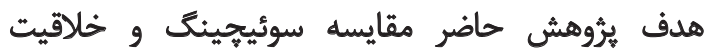

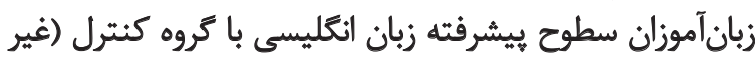

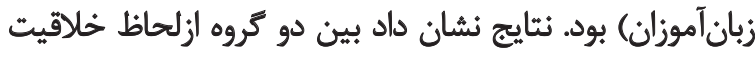

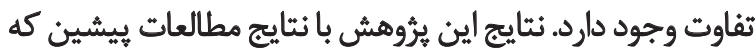

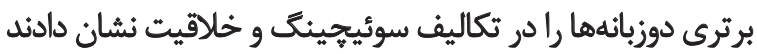

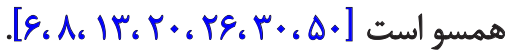

از نتايج بهدستآمده از يُؤوهش حاضر اين مطالب به به ديه

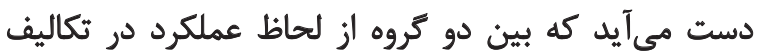

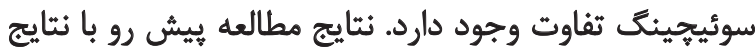

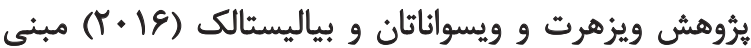

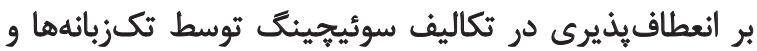

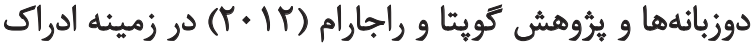

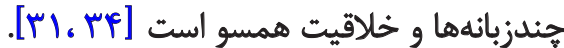
بررسى و تحليل يافتهها نشان مىدهد زبان آموزان در تكاليف

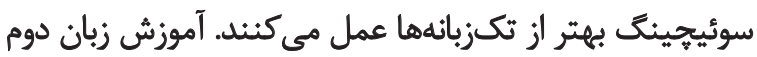

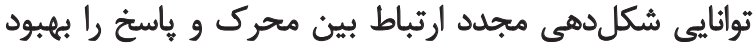

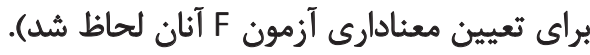

هندمتغيره استفاده شد. براي استفاده از اين روش ابتدا

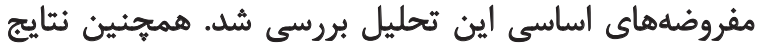

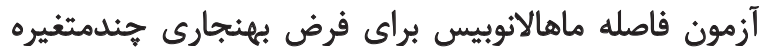

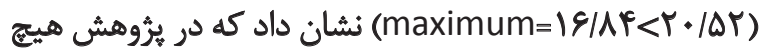

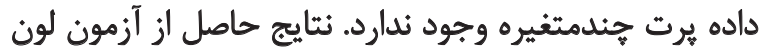

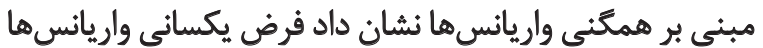

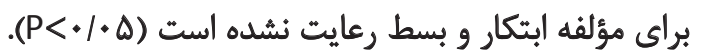
به دليل بالابودن حجم نمونه (بيشتر از • "ام) تخطى از ييشفرض

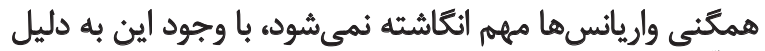

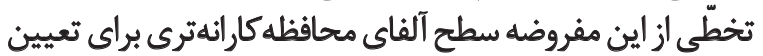

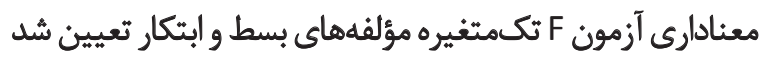

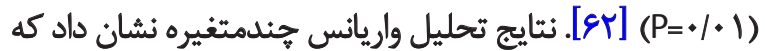

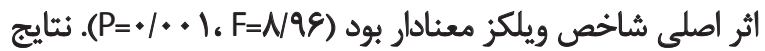

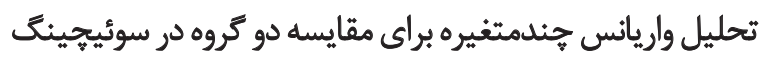

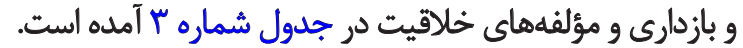
همان طور كه نتايج جدول شماره ب نشان مى مدهد تفاوت

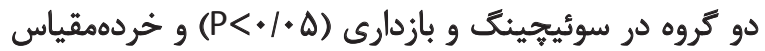

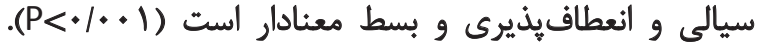

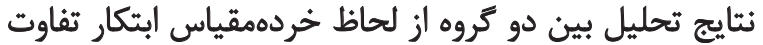

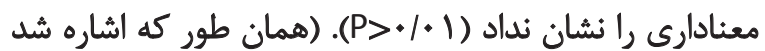
فرض يكسانى واريانسها براى مؤلفه ابتكار و بسط روان رعايت

جدولا. شاخصهاى جمعيت شناختى زبانآموزان و گروه كثترل

\begin{tabular}{|c|c|c|c|c|}
\hline غيرزبانأموز (\%) & زبانأموز (\%) & \multicolumn{2}{|c|}{ سطح } & متغير \\
\hline $\mathbb{T} / N$ & $1 \% \%$ & \multicolumn{2}{|c|}{ ميانكين } & \\
\hline$\cdot / \lambda .$. & • & \multicolumn{2}{|c|}{ انحراف الستأثاراد } & 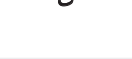 \\
\hline $1 Q(T Q / Y)$ & $\operatorname{reg}(\theta+19)$ & تعداد (درصد) & دختر & \\
\hline$r q(\cdot V / q)$ & 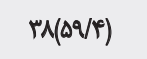 & تعداد (درصد) & يسر & \\
\hline$\|(r V / g)$ & $r(f / \pi)$ & تعداد (درصد) & زير دييلم & \\
\hline $\operatorname{lv}(\pi / / T)$ & $r \cdot(r / T)$ & تعداد (درصد) & دييلم & \\
\hline$F(V / A)$ & $g(q / q)$ & تعداد (درصد) & فوقدييلم & \\
\hline $\operatorname{lV}(\pi / T)$ & $I F(Y \Delta)$ & تعداد (درصد) & ليسأس & تحصيلات يدر \\
\hline$r(r / q)$ & $\operatorname{Ir}(N A)$ & تعداد (درصد) & فوقليسانس & \\
\hline$+(\cdot)$ & $g(q / F)$ & تعداد (درصد) & دكترا & \\
\hline$\| r(\pi / \Delta)$ & $P(g / N)$ & تعداد (درصد) & زير دييلم & \\
\hline$r \cdot(r q / r)$ & MINT/A) & تعداد (درصد) & دييلم & \\
\hline$r(r / q)$ & $\Delta(N / A)$ & تعداد (درصد) & فوقدييلم & حصلات تماد \\
\hline $1 Q(T / / 4)$ & IMPNI) & تعداد (درصد) & ليسانس & 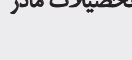 \\
\hline$r(r / q)$ & $I r(r \cdot / r)$ & تعداد (درصد) & فوقليسانس & \\
\hline$\cdot(\cdot)$ & $r(N)$ & تعداد (درصد) & دكترا & \\
\hline
\end{tabular}




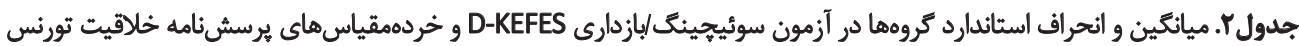

\begin{tabular}{|c|c|c|c|}
\hline انحراف استاندارد & مياكين & كروه & هوزه \\
\hline $898 / 11$ & IEAT/YE & زبان آموز & زمان واكتش در سوئيجينكى \\
\hline$M T / . r$ & $198 / T F$ & غيرزبان آموز & \\
\hline \multirow[t]{2}{*}{ P/RE } & $r I / 0$ & زبان آموز & \\
\hline & & & سيالى س \\
\hline$\Delta / r$ & $1 E M$ & غيرزيان آموز & \\
\hline \multirow[t]{2}{*}{$F / M$} & $T / / N^{C}$ & زبانآموز & \\
\hline & & & انعطاف هيذيرى \\
\hline$\Delta / \Delta H^{\prime \prime}$ & IV/ar & غيرزبان آموز & \\
\hline \multirow[t]{2}{*}{ P/FA } & |W9| & زبان أموز & \\
\hline & & & ابتكار \\
\hline$\Delta / / P$ & $18 / N$ & غيرزيان آموز & \\
\hline \multirow[t]{2}{*}{$r / 8}$. & $r / v q$ & زبان آموز & \\
\hline & & & بسط \\
\hline$F / \Delta A$ & WAF & غيرزبانأموز & \\
\hline
\end{tabular}

توانبخننى

جدول r. نتّايج تحليل واريائس (MANOVA) نمهاى زبان آموزان و غيرزبان آموزان در آزمون سوئيجيئك لبازدارى D-KEFES وخردهمقياس هاى خلاقيت تورنس

\begin{tabular}{|c|c|c|c|c|c|}
\hline سطح معنى قارى & $\mathbf{F}$ & مياتكين مجذورات & df & مجموع مجذورات & خرده مقياس هاي خملاقيت \\
\hline$+1++1$ & $8 / 19$ & TMUIVIVE & 1 & rMIIVINe & سوئيجينك//بازمارى \\
\hline.$/ .1$ & M/Ma & $\Delta r+/ . r$ & 1 & $\Delta r+l \cdot r$ & سيالى \\
\hline .10 .1 & $M T / F$ & AMT/AT & 1 & ATT/ATA & انعطاف يذيرى \\
\hline 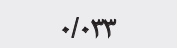 & $\Delta / \cdot V$ & $1 \mathrm{me} / \mathrm{qu}$ & 1 & IrT/u. & ابتكار \\
\hline.$/ .1$ & $\mathscr{I M}$ & MW/TA & 1 & rW/rat & ل بسط \\
\hline
\end{tabular}

عملكرد برتر دوزبانهها را در تسلط و ابتكار كزارش كرديند

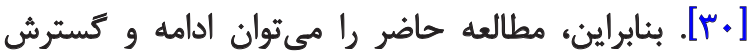

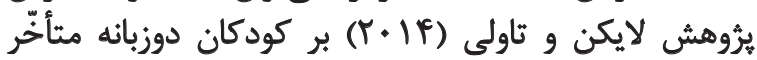

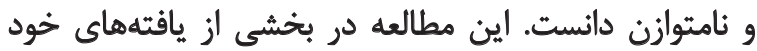

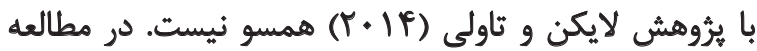

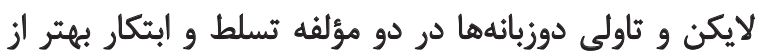

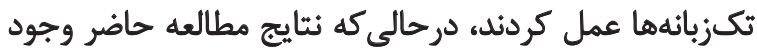

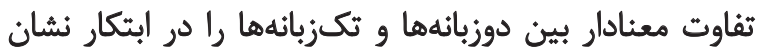

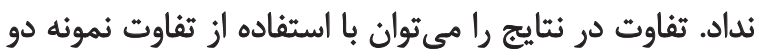
مطالعه در نوع دوزبانكى و سن و دوش روش اندازهكيرى تبيين كرد.

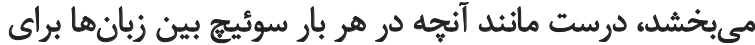

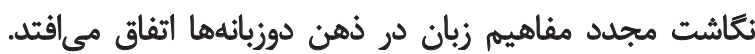

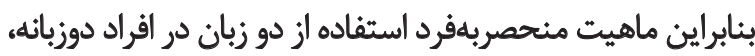

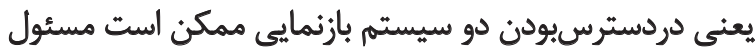

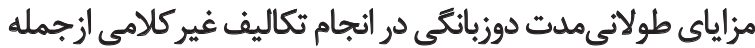

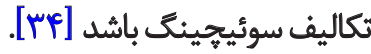

همجنين نتايج نشان داد فراكيرى زبان در مؤسيسات آموزش

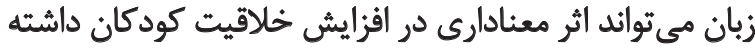

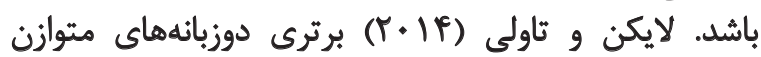

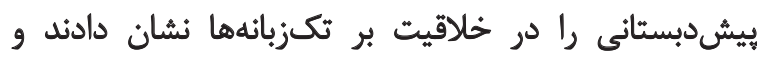


فرهنكى و اقتصادى شركت كنيدكان هر دو كروهاز آموزشكاههاي

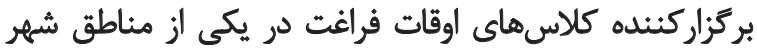

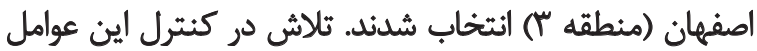

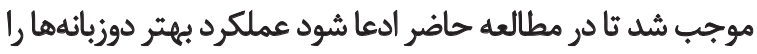
مى توان به فراكيرى زبان دوم از طريق آموزش رسمى نسبت دادي داد.

\section{نتيجهاكيرى}

بر اساس يافتههاى اين يُروهش مي توان نتيجه كرفت فراكيرى

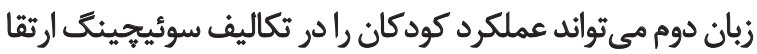

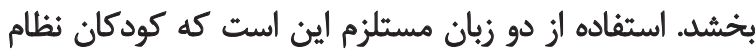

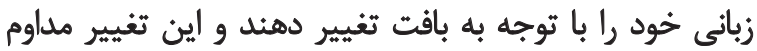

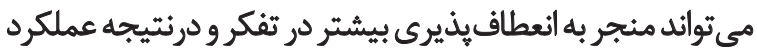

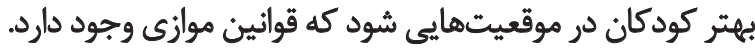

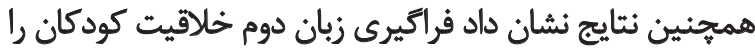

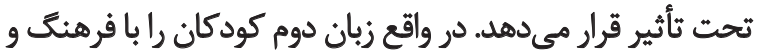

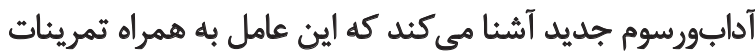

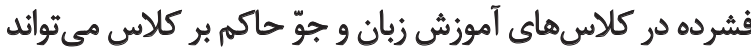

موجب افزايش خلاقيت در زبان آموزان شود.

از محدوديتهاى يثروهش مي توان به مقطعىبودن آن آن اشاره

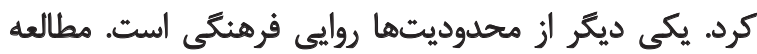

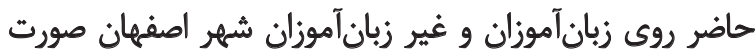

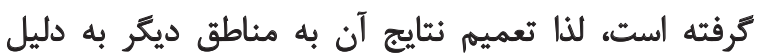

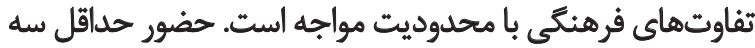

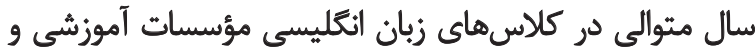

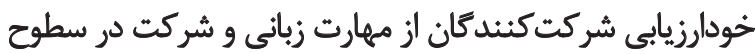

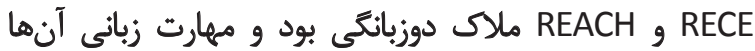

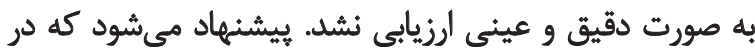

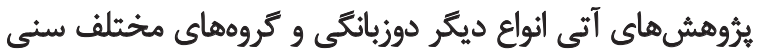

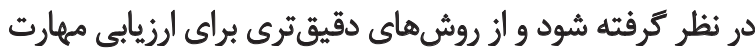

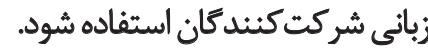

$$
\text { تشكر و قدردانى }
$$

اين مقاله بركرفته از باياننامه ارشد خانم سمير اغضنفريانيور

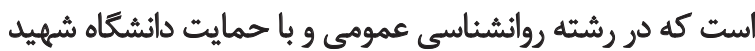

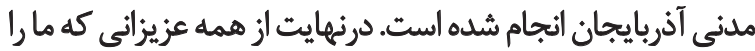

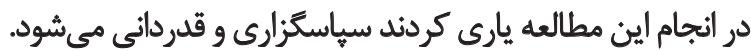

كظريات متعددى براى عملكرد برتر دوزبانهها وجود دارئ بارد.

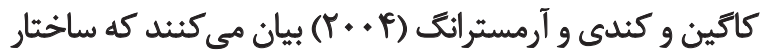

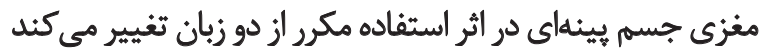

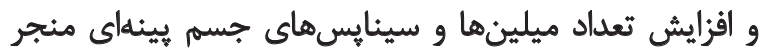

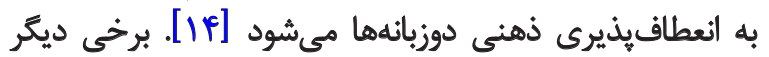

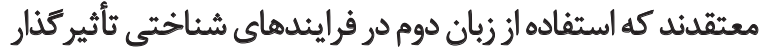

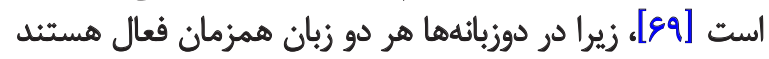

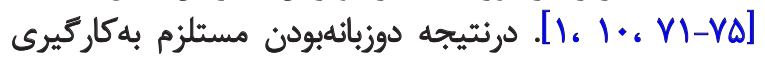

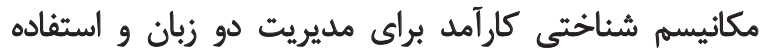

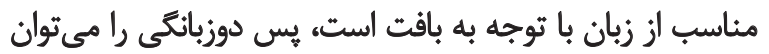

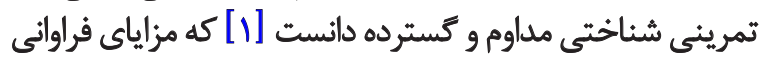

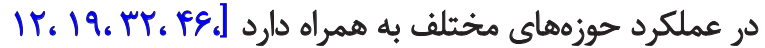

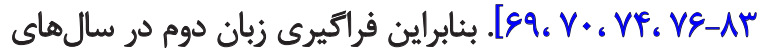

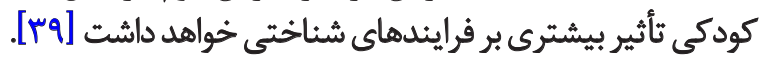
اكرجه فراكيرى و تسلط بر زبان دوم در بافت كلاس درس دئ بافي

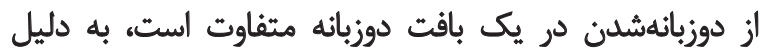

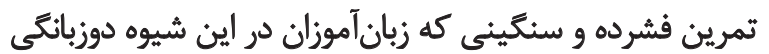

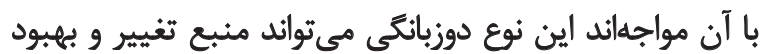

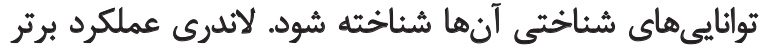

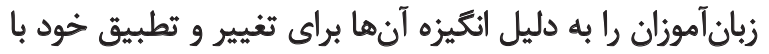

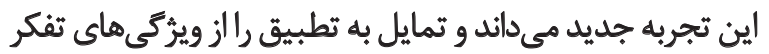

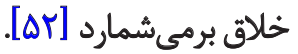

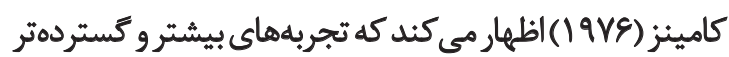

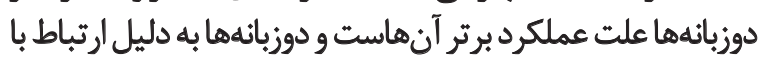

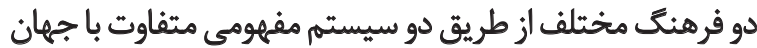

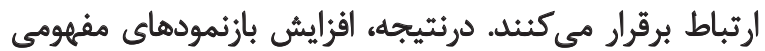

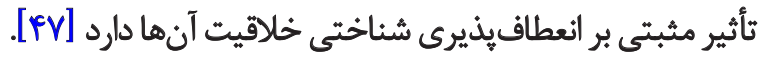

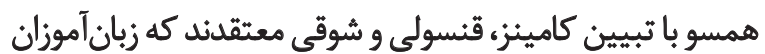

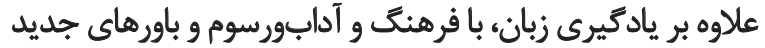

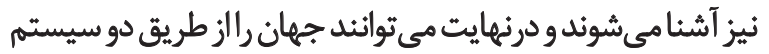

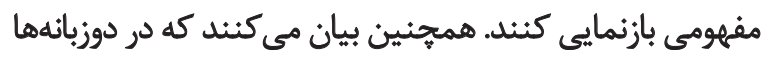

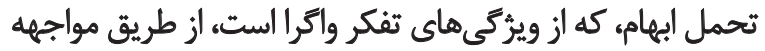

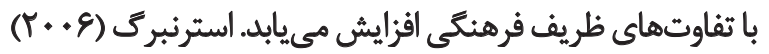

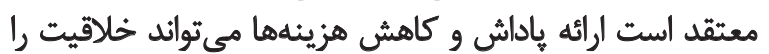

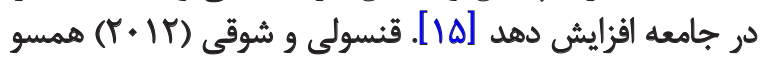

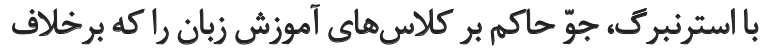

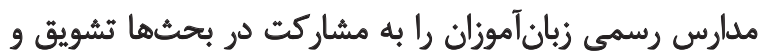

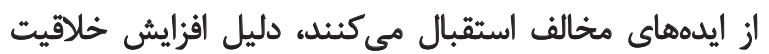

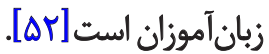

در مطالعه حاضر به منظور كنترل اثر متغيرهاى مزاحم

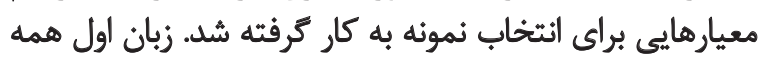

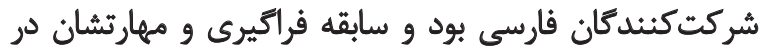

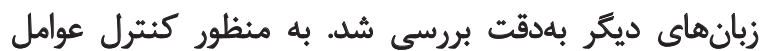




\section{References}

[1] Poarch GJ, Bialystok E. Bilingualism as a model for multitasking. Developmental Review. 2015; 35:113-24. doi: 10.1016/j. dr.2014.12.003

[2] Grosjean F, Li P. The psycholinguistics of bilingualism. Hoboken: John Wiley \& Sons; 2012.

[3] Mohammadi H, Yadegari F, Nili-Pour R, Rahgozar M. [Prevalence of stuttering in Javanroud's bilingual students (Persian)]. Journal of Rehabilitation. 2008; 9(1):43-48

[4] Barac R, Bialystok E, Castro DC, Sanchez M. The cognitive development of young dual language learners: A critical review. Early Childhood Research Quarterly. 2014; 29(4):699-714. doi: 10.1016/j.ecresq.2014.02.003

[5] Adesope OO, Lavin T, Thompson T, Ungerleider C. Pedagogical strategies for teaching literacy to ESL immigrant students: A meta-analysis. British Journal of Educational Psychology. 2011; 81(4):629-53. doi: 10.1111/j.2044-8279.2010.02015.x

[6] Bayanlou A, Azadfallah P, Rasoolzadeh Tabatabaie K. [The development of creative thinking in monolingual and early bilingual children (Persian)]. Social Cognition. 2014; 3(5):100-113.

[7] Beardsmore HB. Multilingualism, cognition and creativity. International CLIL Research Journal. 2008; 3(4):4-18.

[8] Lee H, Kim KH. Can speaking more languages enhance your creativity? Relationship between bilingualism and creative potential among Korean American students with multicultural link. Personality \& Individual Differences. 2011; 50(8):1186-90. doi: 10.1016/j.paid.2011.01.039

[9] Ricciardelli LA. Bilingualism and cognitive development in relation to threshold theory. Journal of Psycholinguistic Research. 1992; 21(4):301-16. doi: 10.1007/bf01067515

[10] Rodriguez-Fornells A, Rotte M, Heinze H-J, Nösselt T, Münte TF. Brain potential and functional MRI evidence for how to handle two languages with one brain. Nature. 2002; 415(6875):10269. doi: $10.1038 / 4151026 a$

[11] Bruck M, Lambert WE, Tucker GR. Cognitive consequences of bilingual schooling: The st. Lambert project through grade six. Linguistics. 1977; 15(187). doi: 10.1515/ling.1977.15.187.13

[12] Kessler C, Quinn ME. Language minority children's linguistic and cognitive creativity. Journal of Multilingual \& Multicultural Development. 1987; 8(1-2):173-86. doi: 10.1080/01434632.1987.9994284

[13] Adesope OO, Lavin T, Thompson T, Ungerleider C. A systematic review and meta-analysis of the cognitive correlates of bilingualism. Review of Educational Research. 2010; 80(2):207-45. doi: $10.3102 / 0034654310368803$

[14] Coggins III PE, Kennedy TJ, Armstrong TA. Bilingual corpus callosum variability. Brain and Language. 2004; 89(1):69-75. doi: $10.1016 /$ s0093-934x(03)00299-2

[15] Bialystok E. Cognitive complexity and attentional control in the bilingual mind. Child Development. 1999; 70(3):636-44. doi: 10.1111/1467-8624.00046
[16] Akbulut Y. Bilingual acquisition and cognitive development in early childhood: Challenges to the research paradigm. İlköğretim Online. 2007; 6(3):422-429.

[17] Bain B. Folate requirement for blast cell transformation in mixed leukocyte cultures. Cellular Immunology. 1975; 15(2):237-45. doi 10.1016/0008-8749(75)90002-7

[18] Kessler C, Quinn ME. Positive effects of bilingualism on science problem-solving abilities. Current issues in bilingual education. Paper presented at: The Annual Georgetown University Round Table on Languages and Linguistics; 1980 June 2-3; Washington, D.C., United States of America.

[19] Bialystok E. Consequences of bilingualism for cognitive development. In: Kroll JF, de Groot AMB, editors. Handbook of Bilingualism: Psycholinguistic Approaches. Oxford: Oxford University Press; 2005

[20] Kharkhurin AV. Bilingual verbal and nonverbal creative behavior. International Journal of Bilingualism. 2010; 14(2):211-26. doi: $10.1177 / 1367006910363060$

[21] Baker C. Foundations of bilingual education and bilingualism. New York: Multilingual Matters; 2011.

[22] Swain M, Cummins J. Bilingualism, cognitive functioning and education. Language Teaching. 1979; 12(1):4. doi: 10.1017/ s0261444800003918

[23] Vaezi S, Zolfaghari AF, Rahimi E. [Cognitive Processing in Bilingual and monolingual Children (Persian)]. Tafakkor va Koodak. 2012; 3(1):119-34.

[24] Paap KR, Johnson HA, Sawi O. Bilingual advantages in executive functioning either do not exist or are restricted to very specific and undetermined circumstances. Cortex. 2015; 69:265-78. doi: 10.1016/j.cortex.2015.04.014

[25] Torrance EP. Torrance tests of creative thinking. Psyctestsdataset [Internet]. 1966 [Cited 1966 June 13]. Available from: http:// dx.doi.org/10.1037/t05532-000

[26] Hommel B, Colzato LS, Fischer R, Christoffels IK. Bilingualism and creativity: Benefits in convergent thinking come with losses in divergent thinking. Frontiers in Psychology. 2011; 2:1-5. Available from: http://dx.doi.org/10.3389/fpsyg.2011.00273

[27] Raina M. Cross-cultural differences. In: Runco MA, Pritzker SR Encyclopedia of Creativity. New York: Academic Press; 1999.

[28] Bott N, Quintin EM, Saggar M, Kienitz E, Royalty A, Hong DWC, et al. Creativity training enhances goal-directed attention and information processing. Thinking Skills \& Creativity. 2014; 13:120-8. doi: 10.1016/j.tsc.2014.03.005

[29] Bogacz R. Optimal decision-making theories: linking neurobiology with behaviour. Trends in Cognitive Sciences. 2007; 11(3):118-25. doi: 10.1016/j.tics.2006.12.006

[30] Leikin M, Tovli E, Malykh S. Bilingualism and creativity in early childhood. Creativity Research Journal. 2014; 3(2):54-63. doi 10.5430/elr.v3n2p54

[31] Gupta N, Rajaram A. Multilingual cognition and creativity. Modern Language Journal. 2012; 22:1-3. 
[32] Bain B. Toward an integration of Piaget and Vygotsky: Bilingual considerations. Linguistics. 1975; 13(160):5-20. doi: 10.1515/ ling:1975.13.160.5

[33] Bahramkhani M, Darvishi N, Keshavarz Z, Dadkhah A. [The camparison of executive functions in normal and autistic children, considering mathematics and reading abilities (Persian)]. Journal of Rehabilitation. 2013; 13:128-35.

[34] Wiseheart M, Viswanathan M, Bialystok E. Flexibility in task switching by monolinguals and bilinguals. Bilingualism: Language and Cognition. 2014; 19(1):141-6. doi: 10.1017/ s1366728914000273

[35] Albakry M, Hancock PH. Code switching in Ahdaf Soueif's the map of love. Language \& Literature. 2008; 17(3):221-34. doi: 10.1177/0963947008092502

[36] Bista K. Factors of Code Switching among Bilingual English Students in the university classroom: A survey. English for Specific Purposes. 2010; 9(29):1-19.

[37] Filippi R, Karaminis T, Thomas MS. Language switching in bilingual production: Empirical data and computational modelling. Bilingualism: Language \& Cognition. 2013; 17(2):294-315. doi: $10.1017 /$ s1366728913000485

[38] Milroy L, Muysken P. One speaker, two languages: Cross-disciplinary perspectives on code-switching. New York: Cambridge University Press; 1995.

[39] Colzato LS, Bajo MT, van den Wildenberg W, Paolieri D, Nieuwenhuis S, La Heij W, et al. How does bilingualism improve executive control? A comparison of active and reactive inhibition mechanisms. Journal of Experimental Psychology: Learning, Memory, \& Cognition. 2008; 34(2):302-12. doi: 10.1037/02787393.34.2.302

[40] Soliman AM. Bilingual advantages of working memory revisited: A latent variable examination. Learning and Individual Differences. 2014; 32:168-77. doi: 10.1016/j.lindif.2014.02.005

[41] Hernandez AE. Language switching in the bilingual brain: What's next? Brain \& language. 2009; 109(2-3):133-40. doi: 10.1016/j.bandl.2008.12.005

[42] Walters J. Bilingualism: The sociopragmatic-psycholinguistic interface. New Jersey: Lawrence Erlbaum; 2014.

[43] Cummins J. Cognitive factors associated with the attainment of intermediate levels of bilingual skills. The Modern Language Journal. 1977; 61(1-2):3-12. doi: 10.2307/325360

[44] Kharkhurin AV. The role of cross-linguistic and cross-cultural experiences in bilinguals' divergent thinking. In: Albertazzi L, Kecskés I, editors. Cognitive Aspects of Bilingualism. Philadelphia: Springer; 2007.

[45] Kharkhurin AV. The effect of linguistic proficiency, age of second language acquisition, and length of exposure to a new cultural environment on bilinguals' divergent thinking. Bilingualism: Language and Cognition. 2008; 11(2):225-43. doi: 10.1017/ S1366728908003398

[46] Kharkhurin AV. The role of bilingualism in creative performance on divergent thinking and invented alien creatures tests.
Journal of Creative Behavior. 2009; 43(1):59-71. doi: 10.1002/ j.2162-6057.2009.tb01306.x

[47] Cummins J. The influence of bilingualism on cognitive growth: A synthesis of research findings and explanatory hypotheses. Toronto: Ontario Institute for Studies in Education; 1976.

[48] Cummins J. Language, power, and pedagogy: Bilingual children in the crossfire. New York: Multilingual Matters; 2000.

[49] Kharkhurin AV. Sociocultural differences in the relationship between bilingualism and creative potential. Journal of Cross-Cultural Psychology. 2010; 41(5-6):776-83. doi: $10.1177 / 0022022110361777$

[50] Leikin M. The effect of bilingualism on creativity: Developmental and educational perspectives. International Journal of Bilingualism. 2012; 17(4):431-47. doi: 10.1177/1367006912438300

[51] Kharkhurin AV. The role of selective attention in bilingual creativity. Creativity Research Journal. 2011; 23(3):239-54. doi: 10.1080/10400419.2011.595979

[52] Ghonsooly B, Showqi S. The effects of foreign language learning on creativity. English Language Teaching. 2012; 5(4):161-67. doi: $10.5539 /$ elt.v5n4p161

[53] Lemmon CR, Goggin JP. The measurement of bilingualism and its relationship to cognitive ability. Applied Psycholinguistics. 1989; 10(2):133-155. doi: 10.1017/s0142716400008493

[54] Hocevar D. Measurement of creativity: Review and critique. Journal of Personality Assessment. 1981; 45(5):450-64. doi: 10.1207/s15327752jpa4505_1

[55] Hamers JF, Blanc M. Bilinguality and bilingualism. Cambridge: Cambridge University Press; 2000.

[56] Birman D, Trickett EJ, Vinokurov A. Acculturation and adaptation of soviet Jewish refugee adolescents: Predictors of adjustment across life domains. American Journal of Community Psychology. 2002; 30(5):585-607. doi: 10.1023/a:1016323213871

[57] Csikszentmihalyi M. Implications of a systems perspective for the study of creativity. In: Sternberg RJ, editor. Handbook of Creativity. Philadelphia: Elsevier; 1999.

[58] Lubart TI. Creativity across cultures. In: Sternberg RJ, editor. Handbook of Creativity. Philadelphia: Elsevier; 1999.

[59] Niu W, Sternberg RJ. Cultural influences on artistic creativity and its evaluation. International Journal of Psychology. 2001; 36(4):225-41. doi: 10.1080/00207590143000036

[60] Skutnabb-Kangas T, Toukomaa P. [Teaching migrant children's mother tongue and learning the language of the host country in the context of the socio-cultural situation of the migrant family (Finnish)]. Tampere: Department of Sociology and Social Psychology at the University of Tampere; 1976.

[61] Mohammadi H, Nili-Pour R, Yadegari F, Karimlou M. [Comparison of linguistic knowledge between students and their healthy peers (Persian)]. Journal of Rehabilitation. 2008; 9(2):47-51.

[62] Lopez EC. Creativity issues concerning linguistically and culturally diverse children. In: Houtz JC, editor. Educational Psychology of Creativity. New Jersey: Hampton Press; 2003. 
[63] Simonton DK. Genius and creativity: Selected papers. Connecticut: Ablex Pub; 1997.

[64] Kharkhurin AV, Samadpour Motalleebi SN. The impact of culture on the creative potential of American, Russian, and Iranian college students. Creativity Research Journal. 2008; 20(4):404-11. doi: 10.1080/10400410802391835

[65] Ghawami H, Raghibi M, Daryadar M. [Impact of English proficiency level on performance in a computerized, English version of Color-Word Interference Test (Persian)]. Paper presented at the $5^{\text {th }}$ International Conference of Cognitive Science. 20 July 2016; Tehran, Iran.

[66] Long EC, Hill J, Luna B, Verhulst B, Clark DB. Disruptive behavior disorders and indicators of disinhibition in adolescents: The BRIEF-SR, anti-saccade task, and D-KEFS color-word interference test. Journal of Adolescence. 2015; 44:182-90. doi: 10.1016/j.adolescence.2015.07.003

[67] Jones Chesters M. D-KEFS validity: An update of the research. Technical Report. Oxford: Pearson Assessment; 2008.

[68] Abedi J. [Creative and new ways to measure it (Persian)]. Psychological Research. 1993; 2:2-10.

[69] Bialystok E, Craik FIM, Luk G. Lexical access in bilinguals: Effects of vocabulary size and executive control. Journal of Neurolinguistics. 2008; 21(6):522-38. doi: 10.1016/j.jneuroling.2007.07.001

[70] Pavlenko A. Bilingualism and thought. In: Kroll J, de Groot A, editors. Handbook of Bilingualism: Psycholinguistic Approaches; Oxford: Oxford University Press; 2005.

[71] Pallant J. SPSS survival manual: A step by step guide to data analysis using the SPSS program [Rezaei A, Persian trans]. $4^{\text {th }}$ ed. Tabriz: Forouzesh; 2010.

[72] Blumenfeld HK, Marian V. Constraints on parallel activation in bilingual spoken language processing: Examining proficiency and lexical status using eye-tracking. Language \& Cognitive Processes. 2007; 22(5):633-60. doi: 10.1080/01690960601000746

[73] Francis WS. Analogical transfer of problem solutions within and between languages in Spanish-English bilinguals. Journal of Memory and Language. 1999; 40(3):301-29. doi: 10.1006/ jmla.1998.2610

[74] Marian V, Spivey M. Bilingual and monolingual processing of competing lexical items. Applied Psycholinguistics. 2003; 24(2):173-193. doi: 10.1017/s0142716403000092

[75] Morales J, Calvo A, Bialystok E. Working memory development in monolingual and bilingual children. Journal of Experimental Child Psychology. 2013; 114(2):187-202. doi: 10.1016/j. jecp.2012.09.002

[76] Schreuder R, Weltens B. The bilingual lexicon. Philadelphia: John Benjamins Pub; 1993.

[77] Abu-Rabia S, Siegel LS. Reading, syntactic, orthographic, and working memory skills of bilingual Arabic-English speaking Canadian children. Journal of Psycholinguistic Research. 2002; 31(6):661-78. PMID: 12599919
[78] Bialystok E, Craik FIM. Cognitive and linguistic processing in the bilingual mind. Current Directions in Psychological Science. 2010; 19(1):19-23. doi: 10.1177/0963721409358571

[79] Bialystok E, Craik FIM, Klein R, Viswanathan M. Bilingualism, aging, and cognitive control: Evidence from the Simon task. Psychology and Aging. 2004; 19(2):290-303. doi: 10.1037/08827974.19.2.290

[80] Duncan SE, De Avila EA. Bilingualism and cognition: Some recent findings. NABE Journal. 1979; 4(1):15-50.

[81] Funk M. Problem solving skills in young yellow-crowned parakeets (Cyanoramphus auriceps). Animal Cognition. 2002; 5(3):167-76. doi: 10.1007/s10071-002-0149-4

[82] Namazi M, Thordardottir E. A working memory, not bilingual advantage, in controlled attention. International Journal of $\mathrm{Bi}$ lingual Education and Bilingualism. 2010; 13(5):597-616. doi 10.1080/13670050.2010.488288

[83] Engel de Abreu PMJ. Working memory in multilingual children: Is there a bilingual effect? Memory. 2011; 19(5):529-37. doi: 10.1080/09658211.2011.590504 
